\title{
Hegel versus Husserl: dos consideraciones sobre la intersubjetividad y la normatividad
}

\author{
Vania Alarcón Castillo \\ Pontificia Universidad Católica del Perú
}

Resumen: El presente artículo compara las consideraciones de Hegel y Husserl sobre la intersubjetividad en relación a la normatividad. Para esto, en una primera parte se discute la descripción de la conciencia del yo y el otro en cada uno de los autores; en la segunda, sus conceptos de libertad; y en la tercera, sus conceptos de normatividad, teleología e historia.

Palabras clave: Husserl, Hegel, intersubjetividad, normatividad, teleología

Abstract: "Hegel versus Husserl: two Accounts of Intersubjectivity and Normativity". This article compares Hegel and Husserl's accounts of intersubjectivity in their relation to normativity. In the first part, each of the authors' descriptions of self consciousness and the consciousness of the other are discussed; in the second part, their concepts of freedom; and in the third, those of normativity, teleology and history.

Keywords: Husserl, Hegel, intersubjectivity, normativity, teleology 
La idea de eticidad o libertad social desarrollada por Hegel es tal vez uno de los hitos teóricos más interesantes de la filosofía moderna, en tanto cuestiona sus presupuestos, advierte sus contradicciones internas y, eventualmente, influye en su propio debilitamiento, aportando, así, al reordenamiento de preocupaciones que marca el paso hacia la contemporaneidad. Entre esos presupuestos destaca el divorcio de lo subjetivo y lo objetivo, el cual, de la mano con la concepción atomista del individuo, acarrea una noción de la libertad que, desde su perspectiva, es unilateral. La propuesta hegeliana, no obstante, tiene sus propios presupuestos, los cuales influyen en su retrato de la normatividad y la historia. Es en este punto donde saltan las críticas más duras contra esta concepción, en particular por su elemento teleológico, que conlleva la afirmación de que la historia consiste en un progreso ascendente de la realización de la conciencia de la libertad (1995, $88 ; 1999, \S 342,490)$.

Si bien muchos filósofos retoman las ideas de Hegel en sus propuestas, la mayoría descarta alguno de sus elementos problemáticos y transforma otros ${ }^{1}$. No obstante, y más allá de lo esperado, uno de los autores en los que se preserva una similar preocupación por la articulación de la racionalidad teórica y práctica, y la relación entre el individuo y la sociedad y su constitución, es Husserl, curiosamente, uno de los filósofos con menos referencias explícitas a Hegel en sus obras (Rizo-Patrón 2012, 267-268). Dentro de la filosofía social y política, Husserl -y la fenomenología en general-, suele ser acusado de un cartesianismo racionalista, individualista, el cual implicaría que no tiene aportes relevantes para estos campos ${ }^{2}$. En efecto, dentro de la enorme obra de Husserl los análisis específicos sobre la libertad y la

1 Por ejemplo, Marx, para quien el concepto de reconocimiento no puede ser realizado dentro del sistema capitalista (Gunn y Wilding 2014, 28) y el Estado no es el punto de superación y solución de los problemas de la sociedad civil (Marx 2010, 25-26, 39); autores como Dilthey o Gadamer, que se deshacen de la noción de espíritu absoluto e incorporan sus elementos dentro del espíritu objetivo (Redding 2011, 212-213) o Honneth, que en diferentes etapas de su desarrollo académico toma y transforma diferentes conceptos hegelianos, a la vez que reniega de otros, por ejemplo, incorpora el "nosotros" (elemento ético) en el mercado ("el nosotros de la acción de la economía del mercado") (2014a, 232 ss.) y excluye su concepto teleológico de progreso (2014b, 825), etc.

2 "If this great tradition of thought <Phenomenology $>$ has one weakness, it is that it assumes things such as autonomy and individuality, and does not pay enough attention to their historical and cultural bases which are best analyzed in the genealogical and critical terms undertaken by Nietzsche. It is perhaps in this respect that phenomenology can often come 
política son escasos. Sin embargo, es notable cierto aire hegeliano en su tratamiento de los problemas de la constitución del individuo y del otro -y de la "comunidad"-. Además, toda su filosofía está atravesada por el tema de la normatividad y su estructura teleológica -debido a su concepción central de la intencionalidad y la evidencia-, a partir de las cuales reflexiona extensamente sobre la historia.

En ambos autores encontramos una pretensión de superar el abismo entre la subjetividad y la objetividad (Staehler 2017, 145), plasmada en la descripción del movimiento del espíritu en la obra de Hegel y en el principio de la correlación y las descripciones genéticas y generativas de Husserl. Sin embargo, debido al mismo método que sigue, Husserl reafirma y retorna siempre al sujeto, ya que es a este al que se le dan todas estas experiencias, quien constituye sus sentidos objetivos (Staehler 2017, 145). Propongo que la teleología abierta descrita por Husserl puede ser un marco normativo atractivo para la idea de libertad social de Hegel, puesto que la historia se concibe como vulnerable a la contingencia y, por tanto, a las crisis (Chu 2005, 243).

Dividiré el trabajo en tres partes: en la primera detallaré las consideraciones de Hegel y Husserl sobre el yo y el otro; en la segunda esbozaré los conceptos de libertad de cada autor, explicando los tipos de relaciones entre individuos que implican, y en la tercera trataré el tema de la normatividad, la teleología y la historia.

\section{$\S 1$. La conciencia del uno y el otro}

Ambos autores sostienen que la experiencia del otro puede ser rastreada a la interioridad, a la autoconciencia (Geniušas 2008, 27). Hegel dedica la segunda parte de la Fenomenología del espíritu a explicar cómo el advenimiento de la autoconciencia está condicionado por la conciencia del otro, en una relación dialéctica o co-constitutiva (Herszenbaun 2017, 83).

across to some philosophers, such as myself, as a bourgeois science" (Ansell-Pearson 2009, 155). 
En cambio, desde sus análisis estáticos, Husserl describe la conciencia del otro en analogía con la autoconciencia, lo cual haría de esta última la experiencia fundamental ${ }^{3}$. Numerosos trabajos privilegian la propuesta de Hegel por encima de la de Husserl, debido a que este incurriría en un individualismo al mantener el lugar privilegiado del "yo-primordial", haciendo de la experiencia del otro algo reducible a la subjetividad (Herszenbaun 2017, 84 , Geniušas 2008, 29 ss.). Sin embargo, creo que esta lectura se restringe a uno de los tres estratos de las descripciones ofrecidas por Husserl, el intermedio: si exploramos textos más allá de las Meditaciones cartesianas, es evidente que reconoce la parcialidad de sus descripciones tempranas y las amplía con sus análisis genéticos y generativos, en los que explora múltiples relaciones y unidades entre el yo y los otros (Steinbock 2003, 192 ss. $)^{4}$. Si bien Hegel parece esbozar mejor la relación co-constitutiva del yo y los otros, la propuesta de Husserl advierte la inmediatez de la experiencia del yo y su distancia inexorable respecto a la inaccesibilidad característica de la experiencia del otro, lo cual eliminaría la posibilidad de que la unidad

3 Reconozco las limitaciones de este punto de partida, puesto que Hegel también usa la analogía como método y, además, Husserl matiza estas descripciones estáticas -donde el primer "yo" es el personal, a partir del cual se constituye el otro (Husserl 1979, §§ 43-49, 152-171)-, en sus análisis genéticos -correspondientes al estrato instintivo (pasivo), donde el primer "yo" es el del otro y el último, el estrato social (Husserl 1979, §§ 49-58, 172-207 [Hua I, 137-162]; Rizo-Patrón 2015, 299 ss.). En efecto, Husserl parte de la autoconciencia en sus análisis estáticos, pero, como revelan los genéticos, esta ya implica a los otros.

4 Para aclarar el origen de estas interpretaciones equivocadas, señalamos que a pesar que las Meditaciones cartesianas -escritas entre 1929 y 1931 - corresponden al periodo genético de la obra de Husserl, este sigue apelando a sus análisis estáticos como "hilos conductores" y, es más, en la quinta meditación (Husserl 1979: §42 ss., 149 ss. [Hua l, 121 ss.]) intenta hacer una descripción estática en la que terminan colándose elementos genéticos, razón por la cual el texto tiene un carácter inacabado -el mismo Husserl lo considera "no publicable" y por eso no se publica en alemán durante su vida (Bernet, Kern y Marbach 1996: 145; Iribarne 1994, 51)-. Sin embargo, justamente este texto es publicado póstumamente como el primer volumen de la Husserliana. Podemos encontrar los desarrollos posteriores de su teoría en Husserliana XIII-XV, y en Husserliana Dokumente II/1 y II/2, textos redactados por Fink en colaboración con Husserl, a los que Husserl añadió múltiples correcciones. Luego de la guerra y de la muerte de Husserl, Fink presentó el texto de Hua Dok II/1 como su tesis de Habilitación, a la cual tituló Sexta meditación cartesiana De ahí que Iribarne sugiera que tanto concentrarse únicamente en la quinta meditación cartesiana como en los análisis estáticos de la intersubjetividad nos deja con una imagen parcial de la teoría de Husserl, y recomienda que tomemos en cuenta ambas consideraciones como interdependientes (Iribarne 1994, 28; (Husserl 1979, §42, n. 37, 149150). Pretendo que esta matización de lo sostenido por Husserl en sus diferentes textos pueda verse en el presente texto, mostrando ciertas aristas de su pensamiento que contradicen las objeciones usuales a su propuesta. 
de la experiencia del yo y del otro caiga en una totalización (Staehler 2017, 184) y denota una actitud alejada de la pretensión de dominio sobre el otro.

\section{a) Hegel: de la conciencia del otro a la autoconciencia}

En la segunda parte de la Fenomenología del espíritu, dedicada al paso de la conciencia al espíritu absoluto, Hegel se enfoca en el problema de la autoconciencia. Esta supone dos aspectos: la conciencia de objetos exteriores y la conciencia de sí misma (Hegel 2008, 107), los cuales generan una contradicción, ya que el segundo solo es posible en tanto la conciencia se diferencia de algo otro (Houlgate 2013, 183). Es decir, para que pueda saberse como autoconciencia, tiene que ser conciencia de algo-otro, o, en otras palabras, su independencia depende de algo diferente: "la autoconciencia es solamente la tautología sin movimiento del yo soy yo; en cuanto que para ella la diferencia no tiene tampoco la figura del ser, no es autoconciencia" $(2008,108)$. Pero tampoco puede haber conciencia sin una autoconciencia: "la conciencia de un otro, de un objeto en general, es, ciertamente, ella misma, necesariamente autoconciencia, ser reflejado en sí, conciencia de sí misma en su ser otro" $(2008,103)$.

Asimismo, Hegel sostiene que la autoconciencia "es, en general, apetencia [Begierde]" $(2008,108)$. El apetito o deseo es considerado una suerte de protoconciencia, un elemento estructurante de la vida biológica animal (Brandom 2011, 31), con alcances para la vida racional. Uno obtiene conciencia teórica de algo, es decir, concibe lo particular como algo general, a partir de su conciencia erótica (erotic awareness) ${ }^{5}$, esto es, sus actitudes prácticas respecto a sus deseos - por ejemplo, cuando un animal hambriento trata un pedazo de carne como alimento procediendo a comerlo (Brandom 2011, 31)-. Así, podemos entender la autoconciencia como un desarrollo de la estructura de la conciencia erótica (Brandom 2011, 34, 38), de manera que un ser autoconciente es un "algo para lo cual las cosas pueden ser algo",

5 Este término es propio de Brandom y no es mencionado por Hegel, mas lo usaré porque es un concepto útil para mi línea de argumentación en este trabajo. 
que asigna significados y puede distinguir entre realidad y apariencia en la experiencia (Brandom 2011, 34, 38) .

Con esto, sin embargo, la autoconciencia perdería su identidad, se fundiría en el otro. De ahí que no solo necesita negar al objeto -con lo cual mantendría su dependencia ${ }^{7}-$, sino que el objeto se pueda negar independientemente a sí mismo, y el único objeto que puede hacer esto es otra autoconciencia (Hegel 2008, 112). El deseo de la autoconciencia solo encuentra una satisfacción momentánea en las cosas, ya que tiene que negarlas (consumirlas) constantemente y, por lo tanto, solo alcanza su satisfacción auténtica con otra autoconciencia $(2008,112)$. Esto se debe a que, mientras los otros-cosas perceptibles son finitos y dependientes, los otros-vivientes son infinitos e independientes, porque generan su propio movimiento y conforman unidades orgánicas (Hegel 2008, 101-102, 109). Es decir, no pueden ser simplemente negados, el deseo se ve obligado a afirmar su independencia $(2008,109)$, negando la diferencia que los separaba (Houlgate 2013 , 183). En efecto, la autoconciencia existe en y para sí únicamente cuando existe para otra, y el concepto de "reconocimiento" refiere a esta relación en la que uno, con la ayuda del otro, adquiere una concepción de sí mismo para-sí, dando cuenta de una revelación prefenomenal del otro, previa a su percepción como tal (Geniušas 2008, 33).

El reconocimiento es, entonces, una actitud normativa recíproca: "la experiencia de lo que el espíritu es, esta sustancia absoluta que, en la perfecta libertad e independencia de su contraposición, es decir, de distintas conciencias de sí que son para sí, es la unidad de las mismas: el yo es el nosotros y el nosotros es el yo" (Hegel 2008, 113). La satisfacción del deseo por reconocimiento requiere reconocer en relaciones simétricas de reconocimiento, es decir, que el sujeto sea reconocido como sujeto por los sujetos que reconoce (Brandom 2011, 39).

Pero además, el reconocimiento es transitivo y, por lo tanto, reflexivo (Brandom 2011, 37). En el acto del reconocimiento de otro sujeto, uno le atribuye

6 Todas las citas textuales de Brandom son traducciones propias.

7 "The very fact that desire feeds on the destruction of the Other means that self-consciousness is dependent upon otherness" (Geniušas 2008, 32). 
autoridad, adoptando la misma actividad de respuesta frente a los mismos objetos, es decir, uno reconoce "a quienesquiera él o ella reconoce" (Brandom 2011, 41-45). Por lo tanto, si el otro lo reconoce, tiene que adoptar la misma actividad frente a sí mismo y, así, auto-reconocerse (Brandom 2011, 49). Entonces, la autoconciencia surge con el reconocimiento: tener una autoconcepción implica concebir a los otros como sujetos capaces de reconocer a otros y ser reconocido como tal por estos. Ambas autoconciencias son independientes y se reconocen como tales y, a la vez, dependen la una de la otra para este reconocimiento (Houlgate 2013, 92). Consecuentemente, la autoconciencia no es inmediata: requiere la mediación del reconocimiento (Staehler 2017, 130). El sujeto y objeto siempre se necesitan correlativamente en la experiencia de la conciencia.

Este esbozo da cuenta de una revelación prefenomenal del otro, previa a la fijación de la atención en él, a su percepción como tal (Geniušas 2008, 33). Esto no significa que el encuentro entre autoconciencias sea pacífico y armonioso, que el reconocimiento sea automáticamente realizado. Brandom sostiene que el paso del plano natural -la conciencia erótica- al espiritual normativo -la conciencia conceptual, de sujetos conocedores y agentes- requiere el advenimiento de la autoconciencia (2011, 33-34). Este movimiento implica un enfrentamiento que pone en jaque la propia vida, una lucha por el reconocimiento, en la búsqueda de superar sus realizaciones parciales (como se muestra en el episodio del señor y siervo). Esto se debe a que cada conciencia quiere ser reconocida unilateralmente, aunque esto sea imposible: necesitan humillarse en su unilateralidad y reconciliarse en un punto superior (Hegel 2008, 391-392; Staehler 2017, 133, 205).

Ahora, desde la Fenomenología y la perspectiva del espíritu subjetivo, se trata la conciencia y reconocimiento del otro, mas, para tratar el problema de la intersubjetividad como tal, hace falta pasar al espíritu objetivo. El análisis de la conciencia, según Hegel, revela que no hay un comienzo incondicionado, un sujeto solipsista ex nihilo (Staehler 2017, 209): la conciencia nunca está sola, sino que es parte de una comunidad que la condiciona y permite su vínculo con la universalidad (Hegel 2008, 111). El otro no resulta una mera extrapolación del yo y el yo no puede autoconstituirse únicamente mediante la percepción o reflexión de sí mismo: necesita la experiencia del 
otro; se trata de una co-constitución (Herszenbaun 2017, 102). El problema de la autoconciencia, que podría aparentar ser meramente epistemológico, termina mostrando así su aspecto ético.

Hegel describirá la relación entre el yo y los otros en tres tipos de unidades o dimensiones de la eticidad (Sittlichkeit), que es el tercer momento del espíritu objetivo que describe la forma de una sociedad animada por una libertad social, tridimensional, en la que la voluntad subjetiva se realiza como racional y universal $(1997, § 513,538-539)$. Estas unidades son la familia, la sociedad civil y el Estado, las cuales discutiré más adelante, concentrándome en este último, donde el reconocimiento y, por lo tanto, la libertad se realizan propiamente.

Ahora, es posible cuestionar el tipo de unidad propuesta por Hegel. Uno podría afirmar que el otro es condición del yo sin necesidad de igualar y unificar sus experiencias. Justamente, la reconciliación podría ser entendida como una supresión de los elementos que la componen (Staehler 2017, $205)^{8}$. Esto tendría la consecuencia, según Ricœur, de anular la responsabilidad de los individuos por sus propios destinos dentro de los colectivos (instituciones) (Herszenbaun 2017, 85). Trataré este temas más adelante, sin embargo, desde ya advierto que la lectura de Ricœur no es del todo justa ${ }^{9}$, debido a que no considera la importancia que Hegel asigna a la moral, entendida como la determinación subjetiva de la voluntad que, si bien es un momento unilateral por ser superado en la eticidad, no puede ser anulado. Esto resalta en su tratamiento de la eticidad griega, la cual critica por su inmediatez y, precisamente, por no dejar espacio para la autonomía. Recordemos que la Aufhebung en la teoría de Hegel es superación y conservación, y no una mera anulación ${ }^{10}$.

8 Esto es cuestionable debido a que la dialéctica de Hegel implica que las determinaciones se mantengan superadas en la unidad (Hegel 1997, §82, 184).

9 Herszenbaun también indica que su lectura se centra en el quinto capítulo de la Fenomenología y pasa por alto el cuarto, donde justamente se discute la intersubjetividad como producto de la interacción de múltiples subjetividades, previa al surgimiento de entidades colectivas como tales $(2017,102,104)$.

10 "No es solo el recorrido de toda la serie de configuraciones unilaterales y limitadas de la conciencia natural -de los distintos conceptos de saber y conceptos de objeto que respectivamente tiene cada figura-, sino que es fundamentalmente un camino transido de negatividad, duda y desesperación. En efecto, en dicho camino se trata de liquidar a cada conciencia 
b) Husserl: de la autoconciencia a la conciencia del otro

Wie die Einzelsubjekte ihre Aktivität auf dem Grund einer dunklen, blinden

Passivität entfalten, so gilt dasselbe auch von der sozialen Aktivität.

Hua XIV, 405

Debido al método fenomenológico que sigue, la consideración de Husserl sobre la relación del yo y el otro parte de la experiencia del sujeto en primera persona (Staehler 2017, 130). Contrario a la mayoría de interpretaciones, no se busca probar la existencia del otro ni "fundamentar la certeza de la experiencia del yo extraño" a partir de la experiencia del sujeto (Herszenbaun $2017,84)$. Más bien, se trata de la pretensión menos ambiciosa de describir nuestra experiencia, en la cual el mundo es dado como una realidad compartida, conformada por otros sujetos que no nos son completamente accesibles (Husserl 2008a, §47, 204 [Hua Vl, 166-167]). Esta es la razón de su orden de presentación en obras como las Meditaciones cartesianas (1979 [Hua I]), en el que se describe primero la experiencia del yo y luego la del otro (\$41, 143 [Hua I, 118]); mas no se trata de un orden de prioridad ontológica. Frente a esto, el punto de partida de Hegel, para quien el otro es una parte constitutiva del yo, no describiría nuestra experiencia del encuentro con el otro, sino el desarrollo conceptual del encuentro entre conciencias ${ }^{11}$. En pocas palabras, mientras la filosofía de Hegel se desarrolla en un nivel conceptual, la de Husserl en uno experiencial, a partir del cual se constituye el conceptual, que es, por lo tanto, secundario.

Sostengo que la propuesta de Husserl no puede ser solipsista porque el sujeto nunca pierde su intencionalidad, es decir, su orientación y tendencia esencial hacia correlatos objetivos trascendentes (Herszenbaun 2017, 90). Para empezar, Husserl lleva a cabo tanto un análisis estático -enfocado en la dimensión activa, racional consciente, y libre, el yo formal- como uno genético -enfocado en la dimensión pasiva, asociativa, instintiva, la historicidad- de la constitución del otro (Rizo-Patrón 2012, 281). En el estático, habla de una reducción a la "esfera de lo mío propio", lo absolutamente propio de

natural con su pretensión unilateral e inmediata de que su 'saber' es saber del absoluto, y que su objeto es el 'en sí' o la verdad" (Rizo-Patrón 2012, 259).

11 "No es que estemos rodeados únicamente de objetos y de pronto encontremos una conciencia diferente por primera vez" (Staehler 2017, 133). 
la subjetividad general (eidos ego) o trascendental, esto es, sus experiencias (nóesis), excluyendo "las efectuaciones constitutivas de la experiencia del extraño" y "todos los modos de conciencia referidos a lo extraño" (Husserl 1979, §44, 157 ss. [Hua l, 126 ss.]). En esta esfera, encontramos la experiencia de la empatía, entendida como la presentificación del otro -y no en el sentido usual del término, esto es, la capacidad de identificarse con alguien y su experiencia o "ponerse en su lugar"-. Para describirla, Husserl hace un paralelo con el recuerdo (1979, §54, 185-186 [Hua I, 147-148]): ambas incluyen una co-presencia, sea del yo-pasado, sea del yo-ajeno, respectivamente, se trata de algo traído a presencia que se mantiene inaccesible (2013, § 99, 327 [Hua III/1, 209]). En el caso del recuerdo, el sujeto hace una síntesis en la que identifica, diacrónicamente, ese yo-pasado con el yo-actual (y futuro), y a sí mismo como yo-duradero a través de la multiplicidad de sus vivencias que pertenecen al mismo flujo o corriente. En el caso de la empatía, el otro, que desde la reducción es visto como un fenómeno, es identificado, sincrónicamente, como un otro-yo, es decir, una subjetividad trascendental que, como tal, pertenece a otra corriente o flujo de vivencias (Husserl 2008a, §54b, 226 [Hua Vl, 210]). Sin embargo, esta "esfera" es un concepto abstracto y provisional, una herramienta útil que ha de superarse y complementarse (Staehler 2017, 137).

En sus trabajos posteriores, Husserl agrega un análisis genético, donde se suspende la experiencia de la empatía ${ }^{12}$ y se habla de una reducción a la esfera solipsista, en este caso, lo absolutamente propio del sujeto concreto (yo personal, mónada), esto es, su soma (Leib) dentro de un campo perceptivo (Husserl 2005, §42, 200-201). Desde este análisis, los otros, inicialmente dados como meros cuerpos físicos (Körper), nos hacen notar en su comportamiento físico que ellos también tienen una "esfera de lo mío propio" ajena, es decir, que también son somas (Leib), ya que experimentamos el autogobierno sobre su movimiento, motivando aquella transferencia de sentido, asociativa y pasiva (Husserl 2005, §45, 205 [Hua IV, 164]). Lo que diferencia al cuerpo del otro sujeto es que "está 'ahí' de tal manera que yo puedo estar ahí posteriormente e incluso me puedo imaginar estando ahí

12 El propósito de esta suspensión es describir la génesis pasiva de la empatía -más profunda que la activa-, ya que esta no es innata, sino que se autoconstituye pasivamente. 
ahora mismo, pero no puedo estar realmente 'ahí' ahora porque 'ahí' está el otro" (Staehler 2017, 139; Husserl 1979, §54, 187 [Hua I, 148]; 2005, §46, 209 [Hua IV, 168]). Lo que se mantiene inaccesible es la conciencia del otro, su flujo de vivencias e historia personal (Herszenbaun 2017, 92). De la misma manera, ser visto por otro me hace darme cuenta que yo también soy otro-yo para el otro, mostrando el carácter recíproco e infinito de la constitución del uno y del otro (Husserl 2005, §46, 210 [Hua IV, 169]). Para Husserl, el yo y el otro son términos relacionales que se constituyen entre sí en tanto se delimitan recíprocamente: "Fände ich in meinem Bewusstsein keine Erscheinungen, die ich als andere Leiber apperzipieren dürfte, so gäbe es für mich kein Du (und somit kein Ich im Gegensatz dazu) und umgekehrt" (Hua XIII, 6). Así, el otro es dado como sujeto y el yo es dado como parte de un nosotros (Husserl 1979, §55, 189 [Hual, 149]) ${ }^{13}$.

Respecto a la experiencia del yo, Husserl explora cuatro niveles de constitución del individuo, además del ego trascendental ${ }^{14}$ (Rizo-Patrón 2012, 281-282): el primer nivel es el del "pre-yo" (Vor-ich), que no es meramente receptivo sino que ya "hace" asociaciones estéticas instintivas (Hua XV, 604) ${ }^{15}$; el segundo es el del "yo primordial" (Ur-ich), como fuente "de la última responsabilidad en las tomas de decisión teóricas y prácticas" (Rizo-Patrón 2012, 282) ; el tercero es el "yo personal individualizado" que tiene un cuerpo y una historia de sedimentaciones; el cuarto es la mónada, la cual tiene un mundo circundante e intersubjetivo que lo determina, es decir, tiene ventanas (Hua XIV, 260; 1979, §33, 121 [102]).

Uno de los puntos de diferencia respecto a Hegel es que este considera la subjetividad como un momento esencial que, no obstante, tiene que

13 "I am aware of myself in a personal and self-referential way through being first of all the beneficiary of a gracious act of attention by Others. In a genetic-constitutional sense I am, first of all, The Other to Others" (Hart 1992, 179)

14 Respecto al yo trascendental, este "se entiende más bien como un yo individual, con un componente esencial natural de instintos originarios y otro esencial racional y libre, responsable de su vida y del sentido del mundo" (Rizo-Patrón 2012, 280).

15 La admisión de la ocurrencia no consciente de asociaciones en el estrato pasivo, fundamentales para la constitución activa, implica que para Husserl, a diferencia de Kant, la pasividad no es una mera afección pasiva o receptividad, sino que es dinámica. La actividad intelectual del sujeto supone, de esta manera, sus procesos asociativos pasivos (Hua XI, 120 ss., 276, 327). 
ser superado (movimiento del espíritu subjetivo al objetivo, y de ahí al absoluto); lo cual, desde la perspectiva de Husserl, sería un abandono del objeto de estudio (Staehler 2017, 145). Sin embargo, todo yo, dice Husserl en la Crisis, porta una intersubjetividad (2008a, §50, 213 [Hua Vl, 175-176]). Señala que la vida personal está marcada por "la necesidad de dejar que la razón personal-individual alcance su actualización como comunitariamente personal" (Husserl 2008a, §73, 305 [Hua Vl, 273]); el individuo tiende a "la comunalización y objetivación de su libertad" (Rizo-Patrón 2012, 282). Es más, afirma que la objetividad está constituida intersubjetivamente (1979, §56, 201 [Hua I, 158]; 2008a, §72, 299) y, por lo tanto, la subjetividad trascendental es, en realidad, una intersubjetividad trascendental, ya que lo reconocido como trascendente supera las daciones particulares, nuestra experiencia subjetiva efectiva (Hua XXIV, 415).

Algunos autores objetan que la transferencia de sentido del yo al otro haría de este último una mera copia del primero, perdiendo la radicalidad de su otredad (Herszenbaun 2017, 94, 107). No obstante, hay que aclarar que para Husserl la experiencia del otro solo puede determinarse como tal a partir de un criterio de identidad normal; por lo tanto, no hay algo así como un otro radicalmente distinto, siempre se da un puente que permite el vínculo y su constitución como tal ${ }^{16}$. El yo y el otro pueden condicionarse mutuamente sin anular la diferencia de sus experiencias correspondientes. La relación entre subjetividad e intersubjetividad es paradójica (Husserl 2008a, §53): la experiencia subjetiva tiene prioridad en el desarrollo individual del ser humano, ya que incluso "las experiencias intersubjetivas son vividas necesariamente desde la primera persona" (Taipale 2012, 50; Husserl 2008a, §73,

16 Los conceptos de normalidad y normatividad están estrechamente ligados en la fenomenología de Husserl y, a su vez, se vinculan con el concepto de tipo. Tanto la experiencia intersubjetiva como la previa (a nivel individual y colectivo, mediante la historia, tradición) contribuyen a la constitución de la normalidad -y, consecuentemente, de la normatividad-, porque los hábitos sedimentados y las anticipaciones que conllevan son correlativos a tipos empíricos, los cuales resultan de las experiencias en las que el sujeto toma posición sobre cómo es el mundo, y a partir de ellos, mediante la variación eidética, se llega a los tipos esenciales o eidos morfológicos (Husserl 2013, § 47, 185 [Hua III/1, 89-90]; Hua XXXVII, § 60 D, 320; 1980, §§ 8-9, 22, 51, 80, 38, 42, 113, 239, 351; Walton 2017: 355, 361; Hugo 2019, p. 309). Así, a través de la "optimización", el proceso selectivo que rastrea los cambios de validez intersubjetivos a nivel histórico, se genera la normatividad (Husserl 2002: 65 [Hua XXVII, 59]; Hua XV: 165; Hua XXXVII: 240, 428; Steinbock 2003: 296). En pocas palabras, la normatividad se constituye a partir de una normalidad, pero la trasciende. 
303 [Hua Vl, 270]), mas no se trata de una secuencia lineal, porque el sujeto nunca es ajeno a lo intersubjetivo, siempre está condicionado en cierta medida y mantiene una relación dinámica con él. Así, la "primera persona”ya es objeto de afecciones pasivas desde el inicio de su vida, por las cuales recibe determinaciones de su entorno, de los sujetos que lo rodean y del mundo, las cuales interactúan con su experiencia activa y las sedimentaciones de la misma (pasividad secundaria).

Según Ricœur, esto dejaría espacio para la responsabilidad individual y la interacción intermonádica dentro del plano intersubjetivo, de manera que los sujetos y colectivos se mantendrían dueños de sus destinos (Ricœur 2001: 277-278; Herszenbaun 2017, 102-103). Husserl Ilama la atención a este tema cuando discute la responsabilidad, la conciencia individual orientada al telos de la humanidad, que trataré en la sección final.

\section{$\S 2$. Libertad}

\section{a) Hegel: la libertad social}

Una de las metas de Hegel en Los principios de la filosofía del derecho es denunciar el divorcio moderno de herencia kantiana entre el derecho y la moral, el abismo entre los fueros externo e interno de la voluntad, uno donde prima la acción y el otro donde prima la intención. Hegel defiende un concepto de libertad social, ya que el espíritu se manifiesta socialmente, objetivamente (Hegel 1997, §385, 437). El concepto de libertad hegeliana se relaciona al reconocimiento, puesto que la autodeterminación es concebida como producto del reconocimiento del otro, de forma que la autonomía está condicionada por lo social.

Este tipo de libertad implicaría una voluntad entendida como "la autodeterminación del yo de ponerse como lo negativo de sí mismo, es decir, de ponerse como determinado, limitado, y al mismo tiempo permanecer consigo, o sea, en su identidad consigo y universalidad, y, en la determinación, unirse sólo consigo mismo" $(1999, \S 7,85)$. Se trata de la unidad de los anteriores momentos abstractos (Hegel 1999, §33, 114), en la que la voluntad 
individual reconoce lo social como realización de su libertad. Así, los fueros "exterior" e "interior" se ven ya no enfrentados, sino reconciliados en una concepción tridimensional (subjetiva, objetiva y absoluta) (Hegel 1999, $\S \S 5-7,81-87)$. Así, las acciones concretas, la sociedad, sus instituciones y normas no se ven como un límite, un obstáculo para nuestra voluntad, sino que son identificadas como producto de la misma (Hegel 1999, §33, 112). Cada momento conserva su legitimidad: insuficientes por separado, hallan su identidad de la identidad y la diferencia en el tercero, el punto de inflexión desde el cual se despliegan y al cual regresan.

La eticidad, el reconocimiento, pasa por tres etapas que corresponden a tres tipos de unidades de individuos: la familia ${ }^{17}$, sociedad civil ${ }^{18}$ y el Estado ${ }^{19}$. Solo en este último, el reconocimiento $y$, por lo tanto, la libertad se realizarán propiamente: los sujetos se reconocen finalmente los unos a los otros como condiciones para su afirmación como sujetos libres, es decir, la realización de su libertad (Honneth 2014a, 61). El reconocimiento, entonces, es el movimiento de la autoconciencia por afirmarse a sí misma en su contradictoria relación con lo otro, frente al cual, finalmente, se descubre dependiente, pero con un margen de agencia, unido por una relación recíproca. Para el individuo, actuar éticamente consiste en considerar el mundo en el que vive, no para seguir mecánicamente sus determinaciones, sino porque sus acciones siempre parten de un contexto, incluso cuando se alejan de este mediante la crítica (Staehler 2017, 189).

17 La familia es descrita como una comunidad armoniosa unida por un vínculo de amor, en la que solo se da un reconocimiento inmediato indiferente a la particularidad (elección libre) de cada miembro (Hegel 1999, §158, 177, 277, 295).

18 En esta, se pierde la armonía de la familia; es el ámbito de los individuos atómicos, el estadio de la diferencia, el reino de necesidades donde las relaciones entre individuos se reducen a lo instrumental (Hegel 1999, §§182, 183, 303-305]).

19 "Cada Estado es un fin por sí mismo. Su preservación externa, su desarrollo interno y formación, siguen un progreso necesario por el cual la racionalidad, justicia y la consolidación de la libertad emergen gradualmente. [Es] un sistema de instituciones -como un sistema [es] la consecuencia y el contenido de las mismas [racionalidad, justicia, libertad], los medios por los cuales los verdaderos intereses son traídos a la conciencia y luchan por obtener su actualidad"; traducción propia ("Every state is an end for itself. Its external preservation, its inner development and formation, follow a necessary progression whereby rationality, justice, and the consolidation of freedom gradually emerge. [It is] a system of institutions - as a system [it is] the consequence and the content of the same [rationality, justice, freedom], the means by which true interests are brought to consciousness and struggle to obtain actuality" (Hegel 1995, 76)). 
Respecto a la libertad, Husserl considera dos dimensiones: un ser libre-de (negativa) y un ser libre-para (positiva) (Hart 1992, 94). Respecto a la primera, sostiene que el ser humano tiene la "peculiaridad esencial de 'actuar' libre y activamente desde sí mismo, desde su yo-centro, en lugar de estar entregado pasivamente a sus impulsos (tendencias, afectos)" (Husserl 2002, 24-25 [Hua XXVII, 23]). Respecto a la segunda, la describe como "una actividad auténticamente 'personal"' en la que "el hombre tiene experiencia [...] piensa, valora, interviene en el mundo circundante" y se orienta hacia una vida en la evidencia (Husserl 2002, 25 [Hua XXVII, 24]).

Para Husserl, "decidir por uno mismo" no implica una independencia respecto al mundo y los otros, sino basarse en la evidencia que experimentamos (Hart 1992, 96), lo cual advierte su carácter esencialmente crítico: "Libertad es [...] una expresión para la facultad y sobre todo para el hábito adquirido de tomar una postura crítica a propósito de lo que, primeramente sin reflexión, se da a la conciencia como verdadero, como valioso, como debiendo ser en sentido práctico" (Husserl 2002, 70 [Hua XXVII, 63]). Una diferencia respecto a Hegel es que Husserl enfatiza que la libertad viene condicionada desde la dimensión pasiva-corporal por el sistema de capacidades motrices, que proveen la limitación fundamental de nuestra voluntad, al restringir el alcance de nuestros órganos de percepción y motrices, relacionados a la acción (Hart 1992, 99).

Respecto a las comunidades de individuos, Husserl diferencia dos tipos: la mera asociación casual, contingente o impuesta por la fuerza y aquella que implica una personalidad de orden superior que la abarca, es decir, que presenta, por analogía, las características de una persona individual y, por lo tanto, activa (Husserl 2002, 23 [Hua XXVII, 22]). Para Husserl, siempre nos encontramos dentro de una unidad de este tipo, una familia, comunidad o Estado (Staehler 2017, 141). Una de las características esenciales de estas comunidades o personalidades de orden superior, que las diferencia de las meras asociaciones fortuitas, es que tienen una historia compartida, un conjunto de hábitos sedimentados, de memorias, expresados en una 
tradición (Hua XIV, 205) ${ }^{20}$. Además, tienen una orientación compartida hacia el futuro, la cual no tiene que ser explícita, sino que funciona como un hábito sedimentado (Staehler 2017, 142). Estas unidades supraindividuales no solo son descriptivas, sino normativas, esto es, describen un ideal práctico (Hart 1992, 267). El ideal final de Husserl de una comunidad del amor o del deber absoluto no se puede realizar perfectamente; es un polo ideal que, sin embargo, es genuino y tiene el efecto práctico de orientar nuestra praxis (Husserl 1987a, §§2, 4-7, 145-148, 150; 1987b, §§7, 8, 138-140 [Hua XIV, 194, 196-198, 175]; Staehler 2017, 197).

Las personalidades de orden superior tienen diferentes grados, según los individuos que agrupan, siendo el último (o más primitivo) la humanidad (Husserl 2002, 64 [Hua XXVII, 58]; Hart 1992, 217)21. Así, siguiendo el principio de la correlación, si cada personalidad de diferente grado tiene un dominio objetivo correspondiente, el mundo será el horizonte objetivo final correlativo a la humanidad (Husserl 1979, §58, 203 [Hua l, 160]). Husserl considera que la constitución de este tipo de comunidades, a las que también llama "mundo-hogar", parte de su contraposición respecto a una cultura o "mundo ajeno"22 (Husserl 1979, §58, 206 [Hua I, 162]; Steinbock $2003,308)$. Nunca califica estas unidades supraindividuales como absolutas, a diferencia de Hegel. Esto se debe a que Husserl no las concibe como algo alejado de sus partes -estas no son meramente accidentales-, sino que se refiere a un nosotros de la vida común (Hart 1992, 264-265).

20 "Von einer sozialen Personalität wäre nur zu sprechen, wenn wir gegenüber den Einzelsubjekten auch von einer Art Ichzentrierung und von einer verharrenden Habitualität der zentrierten Gemeinschaft sprechen können. Ein Verein, eine Stadtmenschheit, vereinigt durch eine Stadtregierung, ein Staatsvolk, einheitlich durch einheitliche Verfassung und Regierung, das sind Beispiele von Personalitäten höherer Ordnung" (Hua XIV, 405).

21 "Por sobre todas las diferencias entre individuos, entre naciones, entre tradiciones en vigor y firmemente arraigadas, se encuentra 'lo que es común a todos' y que responde al título de un 'mundo común de cosas' que se constituye en experiencias intercambiables, de modo que todos pueden entenderse con todos, todos recurrir a una identidad que está a la vista" (Husserl 2002, 84-85 [Hua XXVII, 77]).

22 Husserl usa esta nomenclatura para hacer énfasis en el aspecto histórico constitutivo de los colectivos (Staheler 2017, 143). El término "personalidad de orden superior" parece más adecuado a la perspectiva genética, que explora la intersubjetividad partiendo de una egología, es decir, desde su irradiación desde el yo, mientras que el término "mundo-hogar" es más adecuado a la generativa, la cual enfoca su estudio de la intersubjetividad en sus fuentes históricas en el paso de las generaciones (Steinbock 2003, 292, 303), 
El Estado, para Husserl -a diferencia de Hegel-, es una forma dada del mundo, la encarnación empírica de la conciencia pública, es decir, la consideración de las múltiples perspectivas individuales en forma de servicio estatal (Schuhmann 2009, 86-87, 99; Hart 1992, 389). Desde su perspectiva, el Estado representativo apoyado por Hegel es cuestionable debido a que supone una falsificación del nosotros-común, y funcionaría como un nosotros impuesto "desde arriba", a partir de las representaciones de individuos, en vez de constituirse desde abajo, por los individuos mismos (Hart 1992, 391). El Estado es una unidad de poder, pero eso no la hace automáticamente legítima: "Staat ist eine Einheit durch Macht, durch Herrschaft" (Hua $X V$, 412). Frente a este, la unidad auténtica, constituida por los individuos, implicaría atender directamente las perspectivas de todos (Hart 1992, 395). Sin embargo, Husserl no completa su retrato del Estado ni propone un programa político, aunque Schuhmann señala que, si seguimos sus propuestas de la superación del Estado mediante la comunidad filosófica y científica, se mostraría que, a diferencia de lo propuesto por Hegel, el Estado no es un fin en sí mismo, sino una condición que ayuda a la realización del telos de la humanidad (Schuhmann, 95-98, 100-101; Hart 1992, 403). Asimismo, Hart sugiere que Husserl se orientaría a una propuesta comunitarista, antes que una liberal (Hart 1992, 384, 385).

\section{§3. Normatividad, teleología e historia}

Las propuestas de Husserl y Hegel son alternativas interesantes frente al dilema encarnado por las normas y su estatus como externas e internas a la vez (Redding 2011, 231; Staehler 2017, 198). Esto se debe a que, dentro de sus retratos de la intersubjetividad, ambos se inclinan por revalorizar las normas sedimentadas en hábitos o costumbres, sin que esto implique un carácter estático -ya que resaltan que se trata de un proceso en constante devenir- ni una obediencia mecánica a estas, sino dejando un espacio para la crítica (Hegel 1999, Hua XXXVII, §41 ss., 200 ss.).

El aspecto normativo se relaciona con el teleológico en tanto el ideal que sirve como criterio (norma) también cumple la función de fin hacia el cual 
se tiende en el desarrollo histórico ${ }^{23}$. Ambos autores conciben la historia teleológicamente, en el sentido de orientada hacia fines o metas (Staehler 2017,170 ). Además, los dos asignan un papel especial a la filosofía respecto al espíritu absoluto o de orden superior (Staehler 2017, 173). Esta cumpliría una función para con la humanidad, en la medida en que le permite reflexionar sobre sí misma, aunque en diferente nivel, dándole cierta guía (Husserl 2002, 58 ss. [Hua XXVII, 53 ss.]; Hegel 2008, 80; 1999, 58). Adicionalmente, ambos le dan un privilegio al presente sobre el pasado y futuro; en el caso de Husserl, su concepción abierta de la teleología histórica le permitiría explorar un poco más esa dimensión, pero curiosamente no lo Ilega a hacer (Staehler 2017, 183) ${ }^{24}$.

Una diferencia clave en este punto es aquella entre el espíritu absoluto hegeliano y la personalidad de orden superior. Ambos consideran la religión en su descripción: Hegel la incluye como una dimensión del espíritu absoluto $^{25}$, mientras Husserl la menciona en su descripción de los tipos formales de cultura de la evolución humana, junto a la ciencia y filosofía (Husserl 1987a: §2, 4,6-7, 145, 146, 148-150; 1987b: §8, 139-141; 2002, 65 ss. [Hua XIV, 194, 201-204; Hua XXVII, 59 ss.]). Sin embargo, el concepto hegeliano tiene un componente particular, no se trata de un conglomerado de conciencias individuales, sino que es, desde un inicio, una conciencia común que se reconoce como absoluta a través de su reconocimiento en el desdoblamiento del espíritu: "es el absoluto el que quiere estar con

23 De ahí que estos ideales se planteen como postulados. En el caso de Husserl, esto corresponde a los análisis genéticos, puesto que estos fines (tele) se proyectan constitutivamente como fines a ser realizados paulatinamente.

24 Es conveniente matizar esta afirmación en el caso de Husserl, más allá de lo referido por Staehler. A diferencia del caso de Hegel, donde el presente evidentemente preside sobre el pasado y futuro, en el caso de Husserl, el presente siempre vincula al pasado y futuro o, más específicamente, se articula una tendencia desde el pasado hacia el futuro (donde se muestra el carácter teleológico de la intencionalidad), que hace que todo acto dirigido al presente implique cierta anticipación, de forma que este se "disuelve". Finalmente, aunque la descripción eidética tiende a fijar los sentidos, el tiempo es para Husserl una estructura de la conciencia, es decir, atraviesa la experiencia humana intencional; mientras que, para Hegel, el tiempo es concebido como un momento de la Lógica (de la "Idea en sí y para sí") y, por lo tanto, de la filosofía natural (de la "Idea en sí") desde el cual se desprende el movimiento del espíritu (de la "Idea" en su retorno activo "para sí").

25 "El sí de la reconciliación, en el que los dos yo hacen dejación de su ser contrapuesto [.] es el Dios que se manifiesta en medio de ellos, que se saben como el puro saber" (Hegel 1966, 392). 
nosotros y se reconoce en nosotros" (Staehler 2017, 146). El concepto de Husserl, en cambio, contempla que lo absoluto nunca es completamente dado, lo cual no anula que tengamos una experiencia asintótica del mismo (Husserl 2013, §3, 83, 90-91, 275-276 [Hua III/1, 10, 166-167]).

Esto puede deberse a que, propiamente, Hegel se posiciona en el plano del concepto, en cambio, Husserl, en el de la experiencia: el primero está hablando del encuentro conceptual entre conciencias, mientras que el segundo está describiendo los Yoes y Otros como cuerpos-vivos (Geniušas $2008,35)$. En efecto, uno de los aspectos más característicos de la fenomenología husserliana, que marca su distancia respecto a Hegel, es que reconduce la atención del método hacia la fuente estética del sentido, es decir, la intuición sensible (Rizo-Patrón 2012, 278).

\section{a) Hegel: el espíritu absoluto, la teleología y el progreso}

El tema de la normatividad en la filosofía de Hegel va de la mano con su concepción teleológica de la historia. Retrata la historia como un progreso ascendente, que consiste en el desarrollo absoluto y necesario de la conciencia de la libertad, postula el Estado moderno post Revolución Francesa como fin y, a partir del mismo, da una lectura de las etapas anteriores como su desarrollo parcial (Hegel 1995, 76, 88; 107, Staehler 2017, 170; Gunn y Wilding 2014, 3). El telos es realizable y estático, está prefigurado desde el inicio y en cada etapa (Staehler 2017, 183). Esto ha provocado que su teoría sea tildada de conservadora, porque lo dado es presupuesto como lo mejor y, por lo tanto, no tendría por qué ser cuestionado ni cambiado (Staehler 2017, 170; Honneth 2014a, 10). El futuro, de esa manera, ya estaría contenido en esencia en el presente, lo cual haría que se pierda su esencial carácter sorpresivo (Staehler 2017, 185).

Podríamos responder desde la perspectiva de Hegel que, sin embargo, su pretensión no es ni describir cómo es efectivamente ni cómo debería ser el Estado, sino "el intento de concebir y exponer el Estado como algo en sí mismo racional", lo cual consiste, más bien, "en enseñar cómo él, el universo ético, debe ser conocido" (Hegel 1999, 60). No debemos entender 
su consideración sobre la historia como un cese del movimiento, un estado final, logrado. El retrato de Hegel no describe un proceso extendido en el tiempo objetivo. Ciertamente, rechaza la concepción de un progreso abierto, infinito porque lo propio del espíritu es la eternidad (1971, 131), de forma que no se da en un pasado ni en un futuro, sino que es un estado presente (Hegel 1995, 432). Así, incluso en el Estado moderno la historia sigue, sin por eso sufrir modificaciones que impliquen una superación de la esfera absoluta (Staehler 2017, 172). La sentencia hegeliana: "lo que es racional es real, y lo que es real es racional" $(1999,59)$-es decir, lo racional es efectivo, constituido (wirklich), y viceversa-, no es una afirmación meramente descriptiva, no refiere a la situación presente inmediata, sino a su compleción, el resultado del movimiento dialéctico (Hegel 1997, §6, 106; Rizo-Patrón 2012, 260), es decir, tiene dimensiones normativas que se proyectan más allá de lo dado y que, en el fondo, rescatan el papel efectivo, constituyente de lo racional.

Brandom sostiene que para que una criatura sea autoconsciente su autoconcepción tiene que (1) cumplir una función fundamental en su autoconstitución ${ }^{26}(2011,25)$ y (2) tener una historia -en vez de una naturaleza-, entendida como "una secuencia de auto-transformaciones parcialmente auto-constituyentes, mediadas en cada etapa por sus autoconcepciones, y que culminan en su ser lo que son actualmente" (2011, 25-26). Ambos aspectos van de la mano; ser una criatura histórica requiere autoconcebirse $y$, de esa manera, autoconstituirse como tal -esta dinámica se repite para cualquier característica: somos esencialmente aquello que concebimos como esencial a nuestro ser $(2011,27)-$. Estos dos aspectos implican que el ser autoconsciente puede autotransformarse a partir de su conciencia de sí mismo, de aquello con lo que se identifica -es decir, aquello por lo que está dispuesto a sacrificar lo que es- en una secuencia histórica de auto-transformaciones (de lo que son a aquello con lo que se comprometen a ser-para). Esto daría cuenta de la normatividad a nivel básico individual. Brauer defiende que la racionalidad se orienta, aspira a las instituciones que permitan la realización de la libertad; se trata de un derecho previo,

26 "Cómo algo que es esencialmente autoconsciente aparece a sí mismo es parte de lo que es realmente" (Brandom 2011, 25); "lo que son en-sí mismas está parcialmente determinado en cada etapa por lo que son para-sí mismas" (Brandom 2011, 26). 
efectivo aun en circunstancias en que las condiciones para esta no están dadas $(2014,60,62)$. Este hecho tendría una doble función normativa: en primer lugar, como criterio de legitimidad de órdenes sociales y para juzgar acciones individuales; y, en segundo lugar, como ideal. El Espíritu absoluto, esto es, el espacio de reflexión que trasciende lo dado actualmente en la sociedad, sería el lugar de constitución de la normatividad ${ }^{27}$ (Brauer 2014, 67). El cambio o paso de una forma histórica a otra es explicado apelando a la "vanguardia de la sociedad", donde se ensayarían "formas de justicia que tarde o temprano servirán de modelo a otras naciones" (Brauer 2014, 67), que guían el cambio y progreso en la conciencia (y realización) de la libertad. De forma paralela a lo planteado por Brandom, el colectivo autoconsciente, reflexivo, puede autotransformarse a partir de aquello con lo que se identifica, aquello por lo que están dispuestas a sacrificar lo que es.

Esto fundamentaría la concepción hegeliana de las normas "como socialmente basadas y evolucionando históricamente en un proceso dentro del cual las normas que, en cierto nivel de desarrollo son implícitas a las prácticas sociales, se hacen progresivamente explícitas y disponibles para la reflexión consciente" (Brandom 2011, 217). Las normas no pueden ser autolegisladas, sino deben ser "inmanentes a la vida social-gobernada-por-reglas a la que el sujeto pertenece" (Redding 2011, 231) ${ }^{28}$, lo cual implica un elemento histórico, tradicional, importante, sin negar el potencial crítico de la autoconciencia.

\section{b) Husserl: lo generativo, la teleología abierta y la crisis}

[...] they <humans $>$-individually and in community - posit value goals for themselves to an increasing extent, correlatively, that they can form for themselves an ever more beautiful and better world. Husserl 2019, 479 [Hua VIII, 257/258]

27 Donde "se confirman y revisan reflexivamente los paradigmas normativos que están presenten en la conciencia colectiva, en la opinión pública, en los debates parlamentarios, en las versiones periodísticas del presente y el pasado reciente, etcétera, así como en la elaboración cognitiva de la experiencia histórica" y se reflexiona sobre las posibilidades normativas "para el futuro en el marco de experiencias que ponen en crisis los modelos disponibles" (Brauer 2014, 67).

28 Todas las citas textuales de Redding son traducciones propias. 
De su lado, la consideración de Husserl de nuestra tendencia práctico-ética hacia la evidencia, completitud, la unidad se encuentra ya en su caracterización general de la intencionalidad -característica esencial de la conciencia como una tendencia a su plenificación- (Husserl 2002, 26 [Hua XXVII, 25]). Esta tendencia describe una teleología abierta, ya que su meta no es dada adecuadamente, sino que es una tarea infinita. Lo único dado adecuadamente es la idea de la experiencia infinita, de manera que el telos debe entenderse como el proceso mismo de plenificación a través de series de confirmaciones y decepciones (Bernet 1976, 129-130). Esto es lo que Husserl describe al esbozar el concepto de "idea" e "idea en sentido kantiano": "Sobre la base de la conciencia ejemplar de esta falta de límites captamos además la 'idea' de las direcciones de infinitud determinadas, y la captamos para cada una de las direcciones del transcurso intuitivo que recorremos" (Husserl 2013: §149, 451 [Hua III/1, 312]). Nuestra orientación no predetermina completamente el proceso teleológico, no implica que nuestras metas sean alcanzadas adecuadamente: "en Husserl la transgresión hacia la infinitud jamás abandona la finitud" (Rizo-Patrón 2012, 274). Antes que considerarla un movimiento lineal de progreso ascendente, Husserl concibe la historia como un a priori universal (Chu 2005, 231): "el movimiento vital del uno-con-otro y del uno-en-el-otro de la formación y sedimentación originarias de sentido" (Husserl 2000, 48 [Hua VI, B III 380,), un proceso vulnerable a la contingencia (Husserl 2008a $\_55,56$ [Hua Vl, 10]). Desde su contexto histórico, en medio de las guerras mundiales, la descripción fenomenológica de Husserl no podía afirmar un progreso teleológico ascendente, sería una traición a la experiencia dada (Staehler 2017, 169).

Husserl justifica esta tendencia práctico-ética hacia fines en el hecho de que (1) somos autoconscientes, podemos examinar nuestra vida; (2) tenemos la capacidad de valorar nuestros actos como buenos o malos, justos o injustos, etc.; y (3) somos libres (Husserl 2002, 25-26 [Hua XXVII, 24-25]). Con base en estas características, señala que tendemos hacia nuestra propia unidad, coherencia (Husserl 2002, 32 [Hua XXVII, 30]), por más que nunca tengamos una intuición transparente y adecuada de nosotros mismos. Esa articulación teleológico-normativa es calificada por Husserl como racional y su dimensión de mayor nivel implica a toda la humanidad (Husserl 2008a, 
$\S 73,308$ [Hua Vl, 275]). Nuestro carácter racional y libre nos hace tener responsabilidad, la cual consiste en reconocer nuestro papel constitutivo de sentido (Husserl 2013, 466 [Hua V, 139]) y, en el terreno ético, específicamente, en hacerse cargo de la vida, individual y colectiva, en vistas de llevar a cabo un proceso teleológico de renovación (Husserl 2002, 34 [Hua $X X V I I, 32])$.

Para Husserl, el elemento intersubjetivo permite salvar el aparente abismo entre subjetividad y objetividad (San Martín 2015, 123), sin que el sentido (en este caso, las normas) sea reducido a uno de estos polos. A diferencia de Hegel, no puede haber perfecta adecuación, un auto-conocimiento absoluto; todas las experiencias tienen un horizonte de fondo que implica que no son completas, siempre apuntan hacia otras, y hacia algo más allá del horizonte, oscuro (Husserl 2013, \$45, 177 [Hua III/1, 84]); toda presentación temporal del absoluto tiene un carácter relativo (Rizo-Patrón 2012, 268). El horizonte, precisamente, es lo que permite una relación entre lo directamente accesible, el hogar, y lo accesible-en-su-inaccesibilidad, lo ajeno, y evita que se caiga en un relativismo (Staehler 2017, 173). Además, no se trata solo de una intersubjetividad en el presente, sino histórica: el mundo nos es dado con sentidos preconstituidos, que aprendemos a través de nuestro desarrollo personal.

\section{§4. Conclusión}

Los elementos discutidos hacen que la propuesta husserliana sea atractiva para las perspectivas críticas, a pesar de haber sido pasada por alto por las mismas. Por un lado, evita el conservadurismo que se le reprocha a Hegel -ya que la teleología es abierta y no parte de una valoración positiva de lo dado, sino que reconoce su estado de crisis (Staehler 2017, 170) que, como tal, no debería ser meramente conservado-. Por otro, no requiere invalidar o anular la orientación a fines, necesaria para justificar la normatividad y proveer los criterios normativos. En efecto, a diferencia de la pretensión científica de Hegel, Husserl se orienta por una crítica de la razón, vinculada al concepto de libertad, de manera similar al planteamiento kantiano, pero 
que, en vez de defender una demarcación de la razón, propone "la unidad y co-pertenencia de todas sus dimensiones -teórica, práctica y valorativa" (Rizo-Patrón 2012, 270; Husserl 2008a, §73, 308 [Hua Vl, 275-276]). A diferencia de Hegel, entonces, la historia no consiste en una realización efectiva de la razón, mas apuntamos a su realización exitosa en su desarrollo, a ser iluminados por ella (Staehler 2017, 175).

Creo que las críticas contra Hegel respecto a su conservadurismo y el potencial totalizante de su concepción de la historia no son del todo justas. Efectivamente, su filosofía de la historia contiene afirmaciones mucho más tajantes sobre el progreso, pero eso no anula sus observaciones normativas y críticas, que esbozan las condiciones para la realización de la libertad -incluidas las institucionales-, sin anular el papel del sujeto. Asimismo, creo que el retrato de Husserl como un filósofo racionalista, de una consideración atomista del individuo, niega gran parte de su trabajo, que abarca análisis genéticos y generativos, que como él mismo advierte, tratan fenómenos más concretos (Hua XIV, 34).

La cercanía con Hegel se hace más evidente cuando nos fijamos en esta producción, que retrata al ser humano como parte de una comunidad, producto de un pasado, y orientado por fines comunes en una tarea infinita y corregible. Creo que el vínculo central entre sus filosofías se expresa bien en la famosa afirmación hegeliana sobre lo real y lo racional. No se trata de una afirmación meramente descriptiva; tiene dimensiones normativas que se proyectan más allá de lo dado y que, en el fondo, rescatan el papel efectivo, constituyente de lo racional. Tampoco implica que el mundo es irracional y una conciencia le da sentido arbitrariamente: experimentamos un mundo con sentido, podemos encontrar sentido en la historia y demás producciones humanas (Husserl 2019, 479). Es precisamente nuestra capacidad de revelar y crear vínculos con sentido entre nosotros -y de criticar cuando estos son violados- lo que orienta la historia, antes que nuestra capacidad de hacer normas explícitas y suscribirnos públicamente a ellas (Steinbock 2014, 275). Me atrevo a sostener que son estas experiencias las que abren los espacios de reflexión y constitución normativa que Hegel ubica en el espíritu absoluto y Husserl en la filosofía. Ambos filósofos, desde 
sus propios métodos y preocupaciones específicas, resaltan y articulan esta esencial dimensión de la experiencia humana.

Recibido: 02/03/2020

Aprobado:13/04/2020

\section{Bibliografía}

Ansell-Pearson, Keith, 2009. Evan Thompson, Mind in Life, Biology, Phenomenology, and the Sciences of Mind. Phenomenology and the Cognitive Sciences 8 (1), 151-158.

Bernet, Rudolf, 1976. Perception as a Teleological Process of Cognition. En: Analecta Husserliana, vol. IX, Part III, ed. Anna-Teresa Tymieniecka. Dordrecht/Boston/ Londres: Reidel, 119-132.

Bernet, Rudolf y otros, 1996. Edmund Husserl Darstellung seines Denkens. Hamburg: Felix Meiner Verlag.

Brandom, Robert B., 2011. The Structure of Desire and Recognition: Self-Consciousness and Self-Constitution. En: Recognition and Social Ontology, eds. Heikki Ikäheimo y Arto Laitinen. Leiden: Brill, 25-52.

Brauer, Daniel, 2014. Libertad y autoconciencia. Acerca de los fundamentos de la normatividad en la filosofía práctica de Hegel. En: Dimensiones de la libertad, ed. Miguel Giusti. Lima/Barcelona: Fondo editorial PUCP/Anthropos, 53-67.

Chu, Mariana, 2005. La historicidad y la tarea de la filosofía en Hegel y Husserl. En: Acta fenomenológica latinoamericana 1. Lima: Fondo Editorial PUCP, 213-244.

Geniušas, Saulis, 2008. Self-Consciousness and Other: Hegel and Husserl. Santalka. Filosofija 16 (3), 27-36.

Gunn, Richard y Adrian Wilding, 2014. Introduction. En: Marx and Recognition. Heathwood: Heathwood Institute and Press.

Hart, James, 1992. The Person and the Common Life, New York: Springer.

Hegel, G. W., 1971. Filosofía de la historia. Traducción de José María Quintana. BarceIona: Ediciones Zeus.

- 1995. Lectures on the Philosophy of World History, vol. I. Oxford: Clarendon Press.

- 1997. Enciclopedia de las ciencias filosóficas en compendio (para uso de sus clases). Edición, introducción y notas de Ramón Valls Plana. Madrid: Alianza Editorial.

- 1999. Principios de la Filosofía del derecho. Traducción de J. L. Vermal. Barcelona: Edhasa. 
- 2008. La fenomenología del espíritu. Traducción de Wenceslao Roces. México D.F.: FCE.

Herszenbaun, Miguel, 2017. La intersubjetividad en las Meditaciones cartesianas y Fenomenología del espíritu. Nuevo Itinerario 12, 82-107.

Honneth, Axel, 1991. Lógica de la Emancipación. El legado filosófico del marxismo. Debats 37, 63-65.

- 1995. The Struggle for Recognition. Traducción de J. Andersons. Cambridge: MIT Press.

- 2010. Work and Recognition. En: Schmidt am Busch, Hans-Christoph y Christopher Zurn, The Philosophy of Recognition. Lexington Books: Londres, 223- 237.

- 2014a. El derecho a la libertad. Traducción de Graciela Calderón. Buenos Aires: Katz.

- 2014b. The Normativity of Ethical Life. Philosophy and Social Criticism 40 (8), 817-826.

Houlgate, Stephen, 2013. Hegel's Phenomenology of Spirit. Londres: Bloomsbury.

Hoyos, Guillermo, 2002. La ética fenomenológica como responsabilidad para la Renovación cultural. En: Husserl, E., Renovación del hombre y de la cultura. Traducción de Agustín Serrano de Haro. Barcelona: Anthropos, VII- XXXIII.

Hugo, Zachary, 2019. La constitución e institución intersubjetiva de normas perceptivas en la fenomenología de Husserl. En: Acta fenomenológica latinoamericana, Vi. Lima: Círculo Latinoamericano de Fenomenología / Pontificia Universidad Católica del Perú, 295-310.

Husserl, Edmund, 1950-2015, Husserliana: Edmund Husserl - Gesammelte Werke. Hasta el 201943 volúmenes (Hua I - XLIII).

- Hua I, 1973. Cartesianische Meditationen und Pariser Vorträge. Editado por Stephan Strasser. La Haya: Martinus Nijhoff.

- 1979. Meditaciones Cartesianas. Traducción de Mario Presas. Madrid: Ediciones Paulinas.

- Hua III/1, 1976a. Ideen zu einer reinen Phänomenologie und phänomenologische Philosophie. Erstes Buch: Allgemeine Einführung in die reine Phänomenologie. Editado por Karl Schuhmann. La Haya: Martinus Nijhoff.

- 2013. Ideas relativas a una fenomenología pura y una filosofía fenomenológica, Libro primero: Introducción general a la fenomenología. Nueva edición y refundición integral de la traducción de J. Gaos por A. Zirión Q. México D.F.: UNAM-FCE.

- Hua IV, 1952. Ideen zu einer reinen Phänomenologie und phänomenologische Philosophie. Zweites Buch: Phänomenologische Untersuchungen zur Konstitution. Editado por Marly Biemel. La Haya: Martinus Nijhoff. 
- 2005. Ideas relativas a una fenomenología pura y una filosofía fenomenológica. Libro Segundo: Investigaciones fenomenológicas sobre la constitución. Traducción de Antonio Zirión Q. México D.F.: UNAM-FCE.

- Hua V, 1971. Ideen zu einer reinen Phänomenologie und phänomenologische Philosophie. Drittes Buch: Die Phänomenologie und die Fundamente der Wissenschaften. Editado por Marly Biemel. La Haya: Martinus Nijhoff. = (Nachwort, 138-165).

- 2013. Ideas relativas a una fenomenología pura y una filosofía fenomenológica, Libro primero: Introducción general a la fenomenología. Nueva edición y refundición integral de la traducción de J. Gaos por A. Zirión Q. México D.F.: UNAM-FCE (Epílogo, 465-492).

- Hua Vl, 1976b. Die Krisis der europäischen Wissenschaften und die transzendentale Phänomenologie. Eine Einleitung in die phänomenologische Philosophie. Editado por Marly Biemel. La Haya: Martinus Nijhoff.

- 2008a. Crisis de las ciencias europeas y la fenomenología trascendental. Traducción de Julia V. Iribarne, Buenos Aires: Prometeo.

- 2019. El origen de la geometría. Traducción de Rosemary Rizo-Patrón y Jorge Arce. Estudios de filosofía 4: 33-54.

- Hua VII, 1956. Erste Philosophie (1923/4). Erste Teil: Kritische Ideengeschichte. Editado por Rudolf Boehm. La Haya: Martinus Nijhoff.

- Hua VIII, 1959. Erste Philosophie (1923/4). Zweiter Teil: Theorie der phänomenologischen Reduktion. Editado por Rudolf Boehm. La Haya: Martinus Nijhoff.

- 2019. First Philosophy. Traducción de Sebastian Luft y Thane M. Naberhaus. New York: Springer.

- Hua IX, 1968. Phänomenologische Psychologie. Vorlesungen Sommersemester 1925. Editado por Walter Biemel. La Haya: Martinus Nijhoff.

- Hua X, 1969. Zur Phänomenologie des inneren Zeitbewussteseins (1893-1917). Editado por Rudolf Boehm. La Haya: Martinus Nijhoff.

- 1959. Fenomenología de la conciencia del tiempo inmanente. Traducción de O.E. Langfelder. Madrid: Nova.

- Hua XI, 1966. Analysen zur passiven Synthesis. Aus Vorlesungs- und Forschungsmanuskripten, 1918-1926. Editado por Margot Fleischer. La Haya: Martinus Nijhoff.

- Hua XIII, 1973a. Zur Phänomenologie der Intersubjektivität. Texte aus dem Nachlass. Erster Teil. 1905-1920. Editado por Iso Kern. La Haya: Martinus Nijhoff.

- Hua XIV, 1973b. Zur Phänomenologie der Intersubjektivität. Texte aus dem Nachlass. Zweiter Teil. 1921-28. Editado por Iso Kern. La Haya: Martinus Nijhoff (Gemeingeist I. - Person, personale Ganze, personale Wirkungsgemeinschaften. Gemeinschaft - Gesellschaft [Tx. 9, 165-184]; Gemeingeist II. - Personale Einheiten höherer Ordnung und ihre Wirkungskorrelate [Tx. 10, 192-204]). 
- 1987a. El espíritu común. Traducción de César Moreno Il. Themata 4: 144-150.

- 1987b. El espíritu común. Traducción de César Moreno I. Themata 4: 133-143.

- HuaXV, 1973c. ZurPhänomenologie der Intersubjektivität. Texte aus dem Nachlass. Dritter Teil. 1929- 35.ed.). Editado por Iso Kern. La Haya: Martinus Nijhoff.

- HuaXXIV, 1984. Einleitung in die Logik und Erkenntnistheorie. Vorlesungen 1906/07. Editado por Ullrich Melle. La Haya: Martinus Nijhoff.

- Hua XXVIII, 1988. Vorlesungen über Ethik und Wertlehre 1908-1914. Editado por Ullrich Melle. Dordrecht: Kluwer Academic Publishers.

- Hua XXVII, 1989. Aufsätze und Vorträge [1922-1937]. Editado por Thomas Nenon y Hans.R. Sepp. Dordrecht: Kluwer Academic Publishers.

- 2002. Renovación del hombre y de la cultura. Traducción de Agustín Serrano de Haro. Barcelona: Anthropos.

- Hua XXXVII, 2004. Einleitung in die Ethik. Vorlesungen Sommersemester 1920 und 1924. Editado por Henning Peucker. Dordrecht: Kluwer Academic Publishers.

- Hua XXXIX, 2008b. Die Lebenswelt. Auslegungen der vorgegebenen Welt und ihrer Konstitution. Texte aus dem Nachlass (1916-1937). Editado por Rochus Sowa. New York: Springer.

- Hua XLII, 2014. Grenzprobleme der Phänomenologie. Analysen des Unbewusstseins und der Instinkte. Metaphysik. Späte Ethik. Editado por Rochus Sowa y Thomas Vongehr. New York: Springer.

Husserl, Edmund. 1977 ss. Husserliana: Husserliana Dokumente.

- Hua Dok II/1, 1988. Fink, Eugen. Cartesianische Meditation. Teil I: Die Idee einer transzendentalen Methodelehre. Editado por G. van Kerckhoven, H. Ebeling \& J. Holl. La Haya: Kluwer Academic Publishers.

- Hua Dok II/2, 1988. Fink, Eugen. Cartesianische Meditation. Teil II: Ergänzungsband. La Haya: Kluwer Academic Publishers.

Husserl, Edmund, 1980. Experiencia y juicio. Traducción de Jas Reuter. México D. F.: UNAM.

Iribarne, Julia V. Husserls Theorie der intersubjecktivität. München/Freiburg: Karl Alber $\mathrm{GmbH}$.

Levinas, Emmanuel, 1987. Time and The Other [and additional essays]. Traducción de Richard Cohen, Pittsburgh: Duquesne University Press.

Marx, Karl, 2010. Crítica de la Filosofía del Estado de Hegel. Traducción de Wenceslao Roces. Madrid: Ediciones Biblioteca Nueva.

Melle, Ulrich, 1997. Husserl's Phenomenology of Willing. En: Phenomenology of Values and Valuing, eds. J. Hart y L. Embree. Dordrecht: Springer, 169-192. 
Redding, Paul, 2011. The Relevance of Hegel's 'Absolute Spirit' to Social Normativity. En: Recognition and Social Ontology, eds. Heikki lkäheimo y Arto Laitinen. Leiden: Brill, 211-238.

Ricœur, Paul, 2001. Del texto a la acción. Traducción de Pablo Corona. México D.F.: Fondo de cultura económica.

Rizo-Patrón, Rosemary, 2012. Hegel: sobre la fenomenología y el espíritu. En: Husserl en diálogo: Lectura y debates. Lima/Bogotá: Pontificia Universidad Católica del Perú/Editorial Siglo del Hombre, 257-285.

- 2015. El exilio del sujeto. Lima/Bogotá: Fondo editorial PUCP/Aulda de Humanidades.

San Martín, Javier, 2002. La estructura del método fenomenológico. Madrid: UNED.

- 2008. La fenomenología de Husserl como utopía de la razón. Madrid: Biblioteca Nueva.

- 2015. La nueva imagen de Husserl. Lecciones de Guanajuato. Madrid: Trotta.

Schuhmann, Karl, 2009. Husserl y lo político. Traducción de Julia Iribarne. Buenos Aires: Prometeo.

Staehler, Tanja, 2017. Hegel and Husserl and the Phenomenology of Historical Worlds. Londres: Rowman \& Littlefield.

Steinbock, Anthony, 2003. Generativity and the Scope of Generative Phenomenology. Welton, Donn, The New Husserl. Bloomington: Indiana University Press, 289-325.

- 2014. Moral Emotions. Illinois: Northwestern University Press.

Taipale, Joona, 2012. Twofold Normality: Husserl and the Normative Relevance of Primordial Constitution. Husserl Studies 28, 49-60.

- 2014. Phenomenology and Embodiment. Husserl and the Constitution of Subjectivity. Evanston: Northwestern University Press.

Walton, Roberto, 2017. Intencionalidad y horizonticidad. En: Perception, Affectivity, and Volition in Husserl's Phenomenology, eds. Roberto Walton, Shigeru Taguchi y Roberto Rubio. Nueva York: Springer, 3-20.

Wehrle, Maren, 2015. Normality and Normativity in Experience. En:, Normativity in Perception, eds. Maxime Doyon y Thiemo Breyer. Hampshire: Palgrave, 128-139

Zahavi, Dan, 2001. Husserl and Transcendental Intersubjectivity. A Response to the Linguistic-Pragmatic Critique. Athens: Ohio University Press. 